\title{
Selective Tau Imaging: Der Stand der Dinge*
}

\author{
Victor L. Villemagne \\ Department of Molecular Imaging and Therapy, Austin Health, Melbourne, Australia; Department of Medicine, Austin Health, \\ University of Melbourne, Melbourne, Australia; and Florey Institute of Neuroscience and Mental Health, University of Melbourne, \\ Melbourne, Australia
}

$\mathbf{S}$ gated proteins. A single aggregated protein can manifest as different phenotypes, and a particular phenotype can be caused by different aggregated proteins. The term tauopathies categorizes neurodegenerative conditions, such as Alzheimer disease (AD), characterized by the pathologic accumulation of tau. Tau is a phosphoprotein whose major role is the stabilization of microtubules, critical for intracellular transport and cytoskeletal support. In $\mathrm{AD}$, tau hyperphosphorylation leads to tau aggregation in the form of intracellular filamentous inclusions termed neurofibrillary tangles, and although the mechanisms leading to tau hyperphosphorylation and aggregation have not been fully elucidated, tau deposition follows a stereotypical neuroanatomic pathway in the brain (1).

Since 2011 (2), a steady stream of selective tau tracers for PET has been developed and evaluated in clinical studies (3-7). Several groups have implemented and applied ${ }^{18} \mathrm{~F}-\mathrm{AV} 1451$ (also known as flortaucipir or T807, the most widely used tau tracer to date), in the evaluation of $\mathrm{AD}$ and non- $\mathrm{AD}$ tauopathies $(8,9)$, reporting a robust difference in tau tracer retention between cognitively normal elderly controls and AD patients (Fig. 1) (7-11), as well as in atypical $\mathrm{AD}$ presentations in which ${ }^{18} \mathrm{~F}-\mathrm{AV} 1451$ regional retentionnot $\mathrm{A} \beta$-amyloid as assessed by $\mathrm{PiB}$ - matched the clinical phenotype (12). Furthermore, ${ }^{18} \mathrm{~F}-\mathrm{AV} 1451$ also correlated with cerebrospinal fluid levels of total and phosphorylated tau (13). Interestingly, most studies are showing that although mesial temporal tau is high irrespective of $A \beta$-amyloid levels, high tau in neocortical regions is associated with high $A \beta$-amyloid, suggesting that detectable cortical $\mathrm{A} \beta$-amyloid precedes detectable cortical tau. Tau imaging studies are showing not only that tau tracer retention follows the known distribution of aggregated tau in the brain (1) but also that it has a close relationship with markers of neuronal injury such as ${ }^{18} \mathrm{~F}$-FDG or cortical gray matter atrophy $(14,15)$.

It is also important to highlight that folded and aggregated tau is a challenging neuroimaging target, characterized by intracellular location, 6 different isoforms whose prevalence and combinationslinked to specific phenotypes-are subjected to multiple posttranslation modifications, in turn leading to heterogeneous ultrastructural conformations of the aggregates that, in the particular

\footnotetext{
Received Aug. 24, 2017; revision accepted Sep. 13, 2017.

For correspondence or reprints contact: Victor L. Villemagne, Department of Molecular Imaging and Therapy, Austin Health, 145 Studley Rd., Melbourne, VIC 3084, Australia.

E-mail: victorlv@unimelb.edu.au

*The State of Things.

Published online Sep. 21, 2017.

COPYRIGHT (C) 2018 by the Society of Nuclear Medicine and Molecular Imaging. DOI: 10.2967/jnumed.117.198325
}

case of $\mathrm{AD}$, are in much lower concentrations than $\mathrm{A} \beta$-amyloid aggregates in the same brain regions (16).

Besides ${ }^{18} \mathrm{~F}-\mathrm{AV} 1415(3,8)$, among the first generation of selective tau tracers for PET is ${ }^{11} \mathrm{C}-\mathrm{PBB} 3$ (4), and the series developed by Tohoku (THK) University in Sendai, Japan, namely ${ }^{18}$ F-THK5317 (17) and ${ }^{18} \mathrm{~F}-\mathrm{THK} 5351$ (Fig. 1) (7). Most of these tau tracers bind to the $3 \mathrm{R} / 4 \mathrm{R}$ tau isoform combination prevalent in $\mathrm{AD}$. At this stage, none of these tracers have been validated against pathologic findings, although Avid Radiopharmaceuticals is near the completion of its ${ }^{18} \mathrm{~F}-\mathrm{AV} 1451$ phase III trial.

Some issues arose when these tracers were first used in clinical studies. Although most AD patients present with both high $\mathrm{A} \beta$ amyloid and high tau $(8,9)$, about $15 \%-20 \%$ of subjects diagnosed with probable $\mathrm{AD}$ and with high $\mathrm{A} \beta$-amyloid in the brain have low levels of cortical tau tracer retention. Moreover, there are discrepancies between the preclinical in vitro profile and the in vivo human PET studies $(18,19)$, as well as some antemortem-postmortem inconsistencies $(20,21)$. These inconsistencies do not apply to the $3 R / 4 R$ tau found in $A D$ but mainly to the straight $4 R$ tau filaments found in progressive supranuclear palsy and corticobasal syndrome. For example, tau imaging studies in progressive supranuclear palsy patients show a distinct pattern of tracer retention in the pallidus, midbrain, and dentate nuclei of the cerebellum $(22,23)$, but postmortem studies on some of these patients failed to show binding of the tracer to these structures despite their presenting the typical progressive supranuclear palsy tau lesions $(20,21)$. Also, these tau tracers present with various degrees of what has been called "off-target" binding; that is, tracer retention in brain areas not known for having tau deposition, such as the basal ganglia or the choroid plexus $(19,21)$. Although some have proposed that these tracers bind to aggregated tau in the choroid plexus (24), others have proposed that these tracers bind to other $\beta$-sheet aggregated proteins such as transthyretin, to pigments such as lipofuscin, or to the filaments constitutive of Biondi bodies (18). Recently, it has been reported that a single oral dose of selegiline blocked about $35 \%$ and $50 \%$ of the ${ }^{18}$ F-THK5351 PET signal in the basal ganglia and cortex, indicating that a substantial percentage of the ${ }^{18} \mathrm{~F}$-THK5351 PET signal is due to binding to monoamine oxidase B (25), likely yielding ${ }^{18}$ F-THK5351 not suitable for selective tau imaging studies.

On the other hand, preclinical and preliminary human studies conducted with second-generation tau tracers suggest they are less or not afflicted by off-target binding. Initial human studies of some second-generation tracers such as ${ }^{18} \mathrm{~F}-\mathrm{RO} 06558948$ have shown less severe off-target binding (26), whereas others such as ${ }^{18} \mathrm{~F}-\mathrm{MK} 6240$ (Fig. 1) or ${ }^{18}$ F-PI2620 have shown no off-target binding so far $(5,6)$.

Despite some still unresolved issues, tau imaging is allowing the assessment of the spatial and temporal pattern of tau deposition and its relation to age, genotype, and cognitive performance, helping 


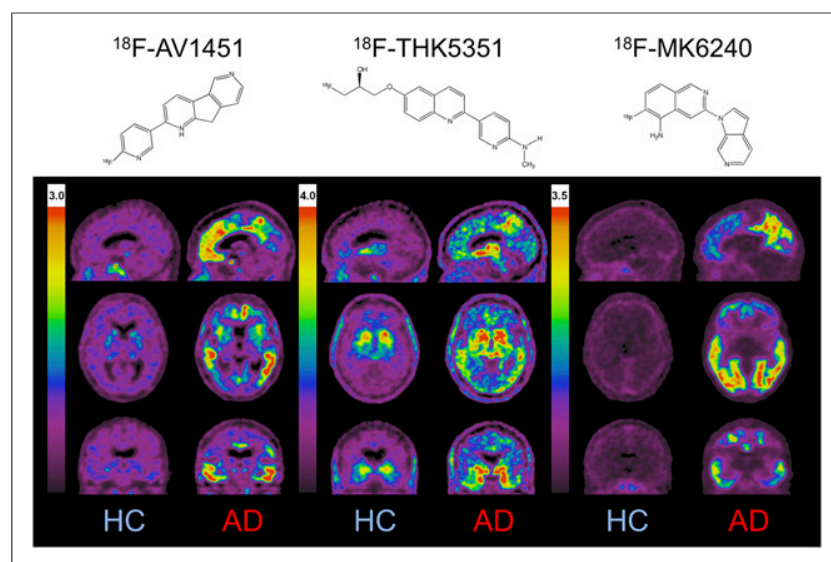

FIGURE 1. (Top) Chemical structures of first-generation $\left({ }^{18} \mathrm{~F}-\mathrm{AV} 1451\right.$ and $\left.{ }^{18} \mathrm{~F}-\mathrm{THK} 5351\right)$ and second-generation ( $\left.{ }^{18} \mathrm{~F}-\mathrm{MK} 6420\right)$ tau tracers. (Bottom) Representative sagittal, transaxial, and coronal (top to bottom) PET images showing robust difference in tracer retention between healthy elderly controls $(\mathrm{HC})$ and $A D$ patients. Off-target binding in basal ganglia, midbrain ( ${ }^{18} \mathrm{~F}-\mathrm{AV} 1451$ and $\left.{ }^{18} \mathrm{~F}-\mathrm{THK} 5351\right)$, and choroid plexus $\left({ }^{18} \mathrm{~F}-\mathrm{AV} 1451\right)$ is observed in PET images obtained with first-generation tracers, where a significant proportion of ${ }^{18} \mathrm{~F}-\mathrm{THK} 5351$ signal is due to binding to monoamine oxidase $\mathrm{B}$. No off-target binding is observed in studies obtained with second-generation ( $\left.{ }^{18} \mathrm{~F}-\mathrm{MK} 6420\right)$ tracer.

elucidate how tau plays a role in sporadic and familial AD, as well as how it relates to $A \beta$ and other imaging and fluid biomarkers. The accurate in vivo identification of tau deposits will allow disease to be staged, prognosis to be determined, and progression to be tracked, eventually leading — when available - to early disease-specific interventions, by optimizing patient selection, providing proof of target engagement, and eventually monitoring therapeutic effectiveness. Tau imaging has opened a unique window to expand our insight into the pathology of $\mathrm{AD}$ and other neurodegenerative conditions.

\section{DISCLOSURE}

No potential conflict of interest relevant to this article was reported.

\section{REFERENCES}

1. Braak H, Braak E. Frequency of stages of Alzheimer-related lesions in different age categories. Neurobiol Aging. 1997;18:351-357.

2. Fodero-Tavoletti MT, Okamura N, Furumoto S, et al. ${ }^{18} \mathrm{~F}-\mathrm{THK} 523$ : a novel in vivo tau imaging ligand for Alzheimer's disease. Brain. 2011;134:1089-1100.

3. Chien DT, Bahri S, Szardenings AK, et al. Early clinical PET imaging results with the novel PHF-tau radioligand [F-18]-T807. J Alzheimers Dis. 2013; 34:457-468.

4. Maruyama M, Shimada H, Suhara T, et al. Imaging of tau pathology in a tauopathy mouse model and in Alzheimer patients compared to normal controls. Neuron. 2013;79:1094-1108.
5. Stephens A, Kroth H, Berndt M, et al. Characterization of novel PET tracers for the assessment of tau pathology in Alzheimer's disease and other tauopathies. Presented at: the $13^{\text {th }}$ International Conference on Alzheimer's and Parkinson's Diseases; March 31, 2017; Vienna, Austria.

6. Walji AM, Hostetler ED, Selnick H, et al. Discovery of 6-(fluoro- $\left.{ }^{18} \mathrm{~F}\right)-3$ $\left({ }^{1} \mathrm{H}\right.$-pyrrolo[2,3-c]pyridin-1-yl)isoquinolin-5-amine ( $\left.\left[{ }^{18} \mathrm{~F}\right]-\mathrm{MK}-6240\right)$ : a positron emission tomography (PET) imaging agent for quantification of neurofibrillary tangles (NFTs). J Med Chem. 2016;59:4778-4789.

7. Okamura N, Furumoto S, Harada R, et al. Characterization of $\left[{ }^{18} \mathrm{~F}\right] \mathrm{THK}-5351$, a novel PET tracer for imaging tau pathology in Alzheimer's disease [abstract]. Eur J Nucl Med Mol Imaging. 2014;41(suppl):S260.

8. Johnson KA, Schultz A, Betensky RA, et al. Tau positron emission tomographic imaging in aging and early Alzheimer disease. Ann Neurol. 2016;79:110-119.

9. Schöll M, Lockhart SN, Schonhaut DR, et al. PET imaging of tau deposition in the aging human brain. Neuron. 2016;89:971-982.

10. Wang L, Benzinger TL, Su Y, et al. Evaluation of tau imaging in staging Alzheimer disease and revealing interactions between beta-amyloid and tauopathy. JAMA Neurol. 2016;73:1070-1077.

11. Cho H, Choi JY, Hwang MS, et al. Tau PET in Alzheimer disease and mild cognitive impairment. Neurology. 2016;87:375-383.

12. Ossenkoppele R, Cohn-Sheehy BI, La Joie R, et al. Atrophy patterns in early clinical stages across distinct phenotypes of Alzheimer's disease. Hum Brain Mapp. 2015;36:4421-4437.

13. Gordon BA, Friedrichsen K, Brier M, et al. The relationship between cerebrospinal fluid markers of Alzheimer pathology and positron emission tomography tau imaging. Brain. 2016;139:2249-2260.

14. Xia C, Makaretz SJ, Caso C, et al. Association of in vivo $\left[{ }^{18} \mathrm{~F}\right] \mathrm{AV}-1451$ tau PET imaging results with cortical atrophy and symptoms in typical and atypical Alzheimer disease. JAMA Neurol. 2017;74:427-436.

15. Chiotis K, Saint-Aubert L, Rodriguez-Vieitez E, et al. Longitudinal changes of tau PET imaging in relation to hypometabolism in prodromal and Alzheimer's disease dementia. Mol Psychiatry. May 16, 2017 [Epub ahead of print].

16. Villemagne VL, Fodero-Tavoletti MT, Masters CL, Rowe CC. Tau imaging: early progress and future directions. Lancet Neurol. 2015;14:114-124.

17. Chiotis K, Saint-Aubert L, Savitcheva I, et al. Imaging in-vivo tau pathology in Alzheimer's disease with THK5317 PET in a multimodal paradigm. Eur J Nucl Med Mol Imaging. 2016;43:1686-1699.

18. Lowe VJ, Curran G, Fang P, et al. An autoradiographic evaluation of AV-1451 tau PET in dementia. Acta Neuropathol Commun. 2016;4:58.

19. Marquié M, Normandin MD, Vanderburg CR, et al. Validating novel tau positron emission tomography tracer [F-18]-AV-1451 (T807) on postmortem brain tissue. Ann Neurol. 2015;78:787-800.

20. Marquié M, Siao Tick Chong M, Anton-Fernandez A, et al. [F-18]-AV-1451 binding correlates with postmortem neurofibrillary tangle Braak staging. Acta Neuropathol (Berl). June 13, 2017 [Epub ahead of print].

21. Marquié M, Normandin MD, Meltzer AC, et al. Pathological correlations of [F-18]-AV-1451 imaging in non-Alzheimer tauopathies. Ann Neurol. 2017;81:117128.

22. Ishiki A, Harada $\mathrm{R}$, Okamura $\mathrm{N}$, et al. Tau imaging with $\left[{ }^{18} \mathrm{~F}\right] \mathrm{THK}-5351$ in progressive supranuclear palsy. Eur J Neurol. 2017;24:130-136.

23. Perez-Soriano A, Stoessl AJ. Tau imaging in progressive supranuclear palsy. Mov Disord. 2017;32:91-93.

24. Ikonomovic MD, Abrahamson EE, Price JC, Mathis CA, Klunk WE. [F-18]AV1451 positron emission tomography retention in choroid plexus: more than "offtarget" binding. Ann Neurol. 2016;80:307-308.

25. Ng KP, Pascoal TA, Mathotaarachchi S, et al. Monoamine oxidase B inhibitor, selegiline, reduces ${ }^{18} \mathrm{~F}$-THK5351 uptake in the human brain. Alzheimers Res Ther. 2017;9:25.

26. Gobbi LC, Knust H, Korner M, et al. Identification of three novel radiotracers for imaging aggregated tau in Alzheimer's disease with positron emission tomography. J Med Chem. 2017;60:7350-7370. 